\section{Journey into the deep.}

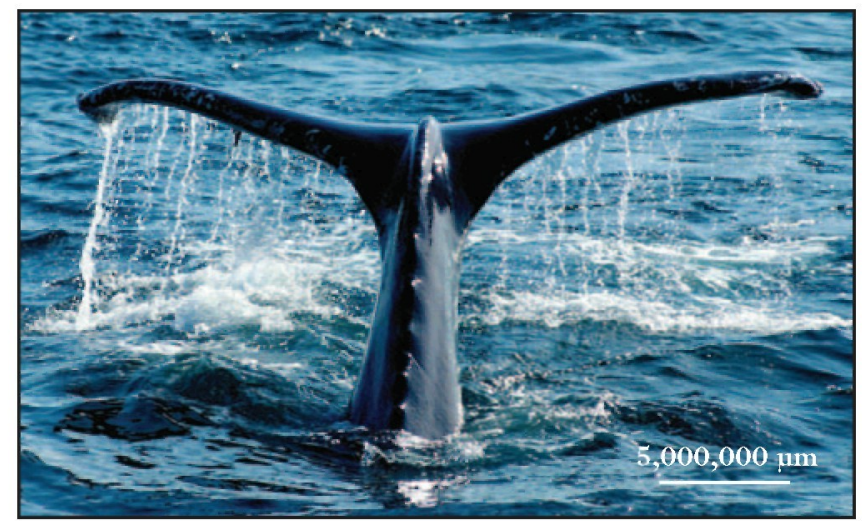

Specimen: Humpback Whale (Genus: Megaptera)

Instrument: The Naked Eye

\section{Journey into the deeper.}

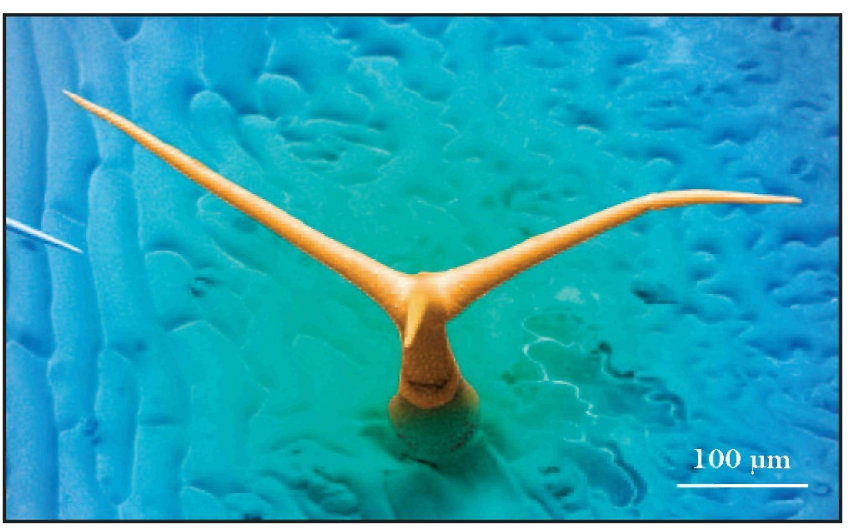

Specimen: Leaf of Arabidopsis Thaliana

Instrument: Hitachi S-3500N SEM
The journey to discover and understand that which cannot be readily seen has often inspired bold exploration.

Hitachi seeks to further that journey by manufacturing leading instrumentation built on a solid foundation of reliability, technology, and technical acumen. Hitachi's nanotechnology tools - a wide variety of ELECTRON MICROSCOPES will empower countless applications to produce stronger, faster, smarter materials that promise to enhance our lives for the better.

\title{
(1) Hitachi High Technologies America, Inc.
}

Electron Microscope Division Headquarters

5100 Franklin Drive

Pleasanton, CA 94588

Sales Phone: (800) 227-8877

Service Phone: (800) 253-3053

www.hitachi-hta.com 
